\title{
Bacterial Leaf Blight on shrunken-2 Sweet Corn
}

\author{
J. K. Pataky, L. J. du Toit, and M. R. Kerns, Department of Crop Sciences, University of Illinois, Urbana 61801
}

\begin{abstract}
Pataky, J. K., du Toit, L. J., and Kerns, M. R. 1997. Bacterial leaf blight on shrunken-2 sweet corn. Plant Dis. 81:1293-1298.

Severity of bacterial leaf blight (BLB, caused by Acidovorax avenae subsp. avenae) on shrunken-2 (sh2) sweet corn hybrids grown in BLB-conducive environments and sporadic outbreaks of BLB in recent years warranted evaluations of the potential for this disease to cause economic losses. BLB symptoms on 122 sh 2 hybrids, 30 sh 2 inbred lines, and 29 crosses of the inbreds and the effects of BLB on yield (ear weight and marketable ears) were evaluated in 1994 and 1996. BLB severity ratings for 122 hybrids averaged 3.3 and 3.7 (on a scale of 1 to 9) in 1994 and 1996, respectively. Hybrid means ranged from 1.8 to 5.5 in 1994 and from 1.9 to 5.7 in 1996, with Bayesian LSD values of 1.20 and 1.22, respectively. There were no differences in BLB ratings among hybrids with yellow, white, or bicolored kernels. Severity of symptoms on $30 \mathrm{sh} 2$ inbreds ranged from 1.5 to 5.7, whereas the reactions of 29 crosses of the inbreds ranged from 1.9 to 5 . The slope coefficient of the regression of crosses on mid-parent values was not different from 1. Thus, mid-parent values gave a good indication of severity of BLB symptoms on crosses, with the exception of a few crosses that tended to be more severely infected than expected based on mid-parent values. Ear weights and number of marketable ears differed among hybrids in the 1994 trial, but there was no significant effect of BLB treatments on either of these measures of yield. In 1996, ear weights in inoculated plots were 10 and $15 \%$ lower than those from noninoculated plots for 2 of 12 hybrids. Despite moderately severe foliar symptoms, BLB did not affect yield substantially in these trials even among hybrids with the most susceptible reactions.
\end{abstract}

Bacterial leaf blight (BLB) of corn ( $\mathrm{Zea}$ mays L.), caused by Acidovorax avenae subsp. avenae (synonym Pseudomonas avenae), is a disease of relatively minor importance in the United States (5), occurring sporadically in many southern and central states $(3,6)$. Infection typically occurs through stomata of leaves in whorls that can harbor large populations of $A$. avenae subsp. avenae and epiphytic bacteria $(2,4)$. During an outbreak of BLB in Florida during the mid-1970s, the bacterium survived for long periods on Vasey's grass (Paspalum urvillei Steud.), a weed commonly found along ditches and roadsides in the sweet corngrowing regions of central Florida (1). Contaminated farm equipment was implicated as a primary means by which the bacterium was disseminated within a field of cv. Gold Cup sweet corn from a reservoir of inoculum found in ditch-bank weeds, including Vasey's grass (1).

When the disease was widespread throughout southern Georgia from 1972 to 1976 , reactions of 35 corn genotypes, including 9 sugary (sul) sweet corn cultivars, were evaluated (6). BLB severity ratings ranged from 1.8 to 9 (on a scale of 1 to 10 ), but

Corresponding author: J. K. Pataky
E-mail: j-pataky@uiuc.edu

Accepted for publication 1 August 1997.

Publication no. D-1997-0828-01R

(C) 1997 The American Phytopathological Society dry matter digestibility did not differ among green and blighted plants. Two sweet corn hybrids, Merit and Apache, were among the cultivars with the most severe BLB symptoms. Three sweet corn cultivars, Bonanza, Tendersweet, and Golden Security, were most resistant to BLB in the trial in 1975. Cv. Gold Cup, evaluated in 1974, was not as susceptible as other hybrids. These sweet corn cultivars are not grown widely today because of the preference for supersweet hybrids with the shrunken-2 (sh2) endosperm mutation.

In 1990, 1992, and 1993, symptoms of BLB were prevalent on many sweet corn hybrids grown in Urbana, IL, in nurseries inoculated with a variety of other pathogens of sweet corn (7). BLB symptoms were severe or moderately severe on 76 of 692 hybrids evaluated in those trials (J. K. Pataky, personal observation). Of 12 severely and 64 moderately severely infected hybrids, 11 and 45, respectively, were $s h 2$, and 30 had white or bicolored kernels. These unusual occurrences of BLB in central Illinois were attributed to exceptionally rainy weather.

BLB should not be prevalent or cause substantial damage on sweet corn in the Midwestern United States under normal weather conditions and typical rainfall. However, severe outbreaks of BLB might occur in central and southern Florida, where nearly all of the sweet corn grown is supersweet, A. avenae subsp. avenae survives in association with ditch-bank and roadside weeds, and the environment is conducive to mois- ture in the whorl. Severe and moderately severe symptoms of BLB were observed in a sweet corn trial near Belle Glade, FL, in 1995 (J. K. Pataky, personal observation). Hybrids with the most severe symptoms included 10 of the $56 \operatorname{sh} 2$ hybrids that had severe or moderately severe BLB symptoms in trials in Illinois from 1990 to 1993.

The severity of BLB symptoms on some of the $s h 2$ hybrids grown in BLB-conducive environments in Florida and the sporadic occurrence of severe BLB symptoms in the Midwestern United States has caused sufficient concern to warrant evaluations of the potential of this disease to cause economic losses. The objectives of this research were to evaluate the severity of BLB symptoms on popular sh2 sweet corn hybrids, to compare BLB symptoms on sh2 hybrids to those on their inbred parents, and to determine the effect of BLB on yield of $s h 2$ hybrids with severe to mild symptoms.

\section{MATERIALS AND METHODS}

Three sets of experiments were done in 1994 and 1996 at the Agronomy/Plant Pathology South Farm, Urbana, IL. The severity of BLB symptoms on 122 sh 2 hybrids and the BLB reactions of 30 sh 2 inbred lines and 29 crosses of the inbreds also were evaluated. The effects of BLB on ear weight and number of marketable ears also were evaluated for 24 hybrids in 1994 and 12 hybrids in 1996.

Hybrid evaluations. BLB symptoms on 65 yellow, 18 white, and 39 bicolored $s h 2$ hybrids from eight commercial seed companies were evaluated in 1994 and 1996. The experimental design was a randomized complete block with three replicates. Each experimental unit was a single row, $3.6 \mathrm{~m}$ long, spaced $76 \mathrm{~cm}$ apart, with about 15 plants per row. Seeds were planted on 17 May 1994 and 20 May 1996.

Strains of A. avenae subsp. avenae, collected in Illinois in 1993 and identified by fatty acid profiles (7), were maintained on greenhouse-grown plants and in 30\% glycerol solutions at $-80^{\circ} \mathrm{C}$. Additional strains were obtained during both years from R. E. Stall (University of Florida, Gainesville). A mixture of all available strains was used as inoculum. Inoculum was prepared in nutrient-broth shake cultures at a room temperature of approximately $22^{\circ} \mathrm{C}$. Cultures were seeded with bacteria approximately $20 \mathrm{~h}$ prior to inoculation. Shake cultures were diluted $1: 20$ with a $0.1 \mathrm{M} \mathrm{NaCl}$ solution immediately before inoculation.

Inoculum was sprayed directly into whorls on 13 and 20 June and 1 and 8 July 1994 
and on 17, 20, 24, and 28 June and 1 July 1996. Whorls held about 2 to $10 \mathrm{ml}$ of inoculum, based on the size of the plant. Plants were at the 3- to 10-leaf stages at the time of inoculation. In 1994, plants were inoculated while moisture from rain or irrigation during the previous evening remained in the whorl.

Rows of plants were rated for severity of BLB symptoms on a scale of 1 to 9: $1=$ chlorotic flecks only; 3 = most symptoms 3 to $10 \mathrm{~cm}$ long and followed leaf veins; $5=$ most symptoms 15 to $30 \mathrm{~cm}$ long; $7=$ most symptoms $50 \mathrm{~cm}$ or longer; and $9=$ symptoms appeared to be systemic on inoculated leaves. Plants were past anthesis in 1994 and at anthesis in 1996 when rows were rated independently by two people on 27 July 1994 and 10 July 1996. Each rater gave each row three ratings to reflect the variability of symptoms within rows. For example, a row would have been rated 3-3-5 if symptoms on two-thirds of the plants were in category 3 and those on one-third were in category 5 .

BLB severity ratings were analyzed by analysis of variance (ANOVA), and WallerDuncan Bayesian LSD (BLSD) values were used to compare hybrids. Groups of hybrids with yellow, white, or bicolored kernels were compared by $t$ tests. Reactions of hybrids to BLB were classified from resistant (R) to susceptible (S), according to BLSD separations of symptom severity. Hybrids with ratings that were not different from the lowest rating but were significantly lower than the trial mean were classified as R. Those that were not different from the trial mean and the lowest rating were classified as moderately resistant (MR). Those that were not different from the highest rating and were significantly greater than the trial mean were classified as S. Those that were not different from the trial mean and the highest rating were classified as moderately susceptible (MS). Those that were different from the highest and lowest ratings and were not different from the trial mean were classified as moderate (M).

Inbreds and crosses. Severity of BLB symptoms on $30 \mathrm{sh} 2$ inbred lines and 29 hybrids derived from crosses of those inbreds were evaluated in 1994 and 1996. The experimental design was a split-plot randomized complete block with five replications. Hybrids or inbreds were assigned to main plots, and subplots were planted with specific genotypes. Each experimental unit was a single row, $3.6 \mathrm{~m}$ long, spaced $76 \mathrm{~cm}$ apart, with about 15 plants per row. Seeds were planted on 17 May 1994 and 20 May 1996. Plants were inoculated, and severity of symptoms was rated as described for the hybrid trials.

Severity of BLB symptoms was analyzed by ANOVA. Inbreds and crosses were compared by Waller-Duncan BLSD values $(k=$ 100). Mid-parent values for crosses were calculated as arithmetic means of the seed and pollen inbred parents. BLB severity ratings for crosses were regressed on midparent values, and differences between these and mid-parent values were calculated.

Yield-loss trials. Effects of BLB on ear weight and percentage of ears acceptable for fresh market were evaluated for $24 \operatorname{sh} 2$ hybrids in 1994 and 12 of the 24 hybrids in 1996. The treatment designs were $24 \times 2$ or $12 \times 2$ factorials of hybrids and BLB treatments: inoculated and noninoculated plots.

Table 1. Severity ratings and disease reactions to bacterial leaf blight (BLB) for 122 shrunken-2 sweet corn hybrids evaluated in 1994 and $1996^{\mathrm{a}}$

\begin{tabular}{|c|c|c|c|c|c|c|c|}
\hline \multirow[b]{2}{*}{ Hybrid } & \multirow{2}{*}{$\begin{array}{c}\text { Kernel } \\
\text { color }^{b}\end{array}$} & \multirow{2}{*}{$\begin{array}{c}\text { Seed } \\
\text { co. }^{\mathrm{c}}\end{array}$} & \multicolumn{2}{|c|}{ Reaction to $B^{2} B^{d}$} & \multicolumn{3}{|c|}{ Severity rating ${ }^{\mathrm{e}}$} \\
\hline & & & 1994 & 1996 & $\bar{x}$ & 1994 & 1996 \\
\hline Jupiter & Y & Sdw & MR & $\mathrm{R}$ & 2.1 & 2.3 & 1.9 \\
\hline Crisp n Sweet 710A & Y & $\mathrm{Cr}$ & MR & $\mathrm{R}$ & 2.1 & 2.3 & 2.0 \\
\hline Summer Sweet 7902 & $\mathrm{~B}$ & $\mathrm{AC}$ & MR & $\mathrm{R}$ & 2.1 & 2.3 & 2.0 \\
\hline BSS 4751 & $\mathrm{~B}$ & $\operatorname{Rog}$ & $\mathrm{R}$ & MR & 2.2 & 1.8 & 2.7 \\
\hline Midship & Y & Sdw & $\mathrm{R}$ & MR & 2.2 & 1.8 & 2.7 \\
\hline Sch 5005 & $\mathrm{Y}$ & IFS & $\mathrm{R}$ & $\mathrm{R}$ & 2.2 & 2.0 & 2.4 \\
\hline Apollo & $\mathrm{Y}$ & Sdw & MR & $\mathrm{R}$ & 2.2 & 2.3 & 2.2 \\
\hline Sch 20777 & $\mathrm{Y}$ & IFS & MR & $\mathrm{R}$ & 2.3 & 2.8 & 1.9 \\
\hline ХРН 3099 & $\mathrm{~W}$ & Asg & MR & $\mathrm{R}$ & 2.4 & 2.5 & 2.3 \\
\hline Crisp n Sweet 711 & $\mathrm{Y}$ & $\mathrm{Cr}$ & MR & MR & 2.5 & 2.3 & 2.8 \\
\hline Starship & $\mathrm{B}$ & Sdw & MR & MR & 2.5 & 2.5 & 2.6 \\
\hline ACX 92 CN $17 \mathrm{M}$ & $\mathrm{Y}$ & $\mathrm{AC}$ & MR & $\mathrm{R}$ & 2.5 & 2.8 & 2.3 \\
\hline Flare & $\mathrm{Y}$ & Rog & MR & $\mathrm{R}$ & 2.6 & 2.8 & 2.4 \\
\hline Summer Sweet 7630 & Y & $\mathrm{AC}$ & MR & MR & 2.7 & 2.5 & 2.9 \\
\hline Eminence & $\mathrm{B}$ & $\mathrm{Cr}$ & M & $\mathrm{R}$ & 2.7 & 3.0 & 2.4 \\
\hline HMX 2346 BS & $\mathrm{B}$ & HM & MR & MR & 2.8 & 2.5 & 3.0 \\
\hline Ultimate & Y & HM & M & MR & 2.8 & 3.0 & 2.6 \\
\hline Cabaret & $\mathrm{B}$ & Asg & $\mathrm{R}$ & M & 2.8 & 2.0 & 3.6 \\
\hline Marvel & $\mathrm{Y}$ & $\mathrm{Cr}$ & $\mathrm{R}$ & M & 2.8 & 2.0 & 3.6 \\
\hline Punchline & $\mathrm{Y}$ & Asg & MR & M & 2.8 & 2.3 & 3.3 \\
\hline HMX 2344 BS & $\mathrm{B}$ & $\mathrm{HM}$ & MR & MR & 2.8 & 2.8 & 2.9 \\
\hline Endeavor & Y & Asg & MR & M & 2.9 & 2.5 & 3.2 \\
\hline Sch 34400 & B & IFS & MR & M & 2.9 & 2.8 & 3.1 \\
\hline Festival & $\mathrm{B}$ & Asg & MR & M & 2.9 & 2.8 & 3.1 \\
\hline Summer Sweet 7631 & $\mathrm{~W}$ & $\mathrm{AC}$ & $\mathrm{R}$ & M & 2.9 & 2.0 & 3.9 \\
\hline Phenomenal & $\mathrm{B}$ & $\mathrm{Cr}$ & M & MR & 3.0 & 3.3 & 2.7 \\
\hline Sweet Magic & W & HM & MR & M & 3.0 & 2.3 & 3.7 \\
\hline Summer Sweet 7210 & Y & $\mathrm{AC}$ & MR & M & 3.0 & 2.3 & 3.8 \\
\hline WSS 4175 & $\mathrm{~W}$ & Rog & MR & M & 3.0 & 2.3 & 3.8 \\
\hline Pro Sweet $314 \mathrm{R}$ & $\mathrm{Y}$ & $\mathrm{AC}$ & M & MR & 3.0 & 3.3 & 2.8 \\
\hline Pro Sweet $415 \mathrm{R}$ & $\mathrm{Y}$ & $\mathrm{AC}$ & M & M & 3.1 & 3.0 & 3.1 \\
\hline Crisp n Sweet 710 & $\mathrm{Y}$ & $\mathrm{Cr}$ & MR & M & 3.1 & 2.3 & 3.9 \\
\hline XPH 3092 & W & Asg & MR & M & 3.1 & 2.3 & 3.9 \\
\hline Golden Gourmet & Y & $\mathrm{HM}$ & MR & M & 3.1 & 2.8 & 3.4 \\
\hline Day Star & $\mathrm{Y}$ & HM & MR & M & 3.1 & 2.8 & 3.4 \\
\hline Pinnacle & $\mathrm{Y}$ & HM & M & M & 3.1 & 3.0 & 3.2 \\
\hline WSS 5463 & W & $\operatorname{Rog}$ & M & M & 3.1 & 3.0 & 3.2 \\
\hline Maxim & $\mathrm{Y}$ & $\mathrm{HM}$ & M & MR & 3.2 & 3.8 & 2.6 \\
\hline GSS 4606 & $\mathrm{Y}$ & $\operatorname{Rog}$ & MR & M & 3.2 & 2.8 & 3.6 \\
\hline Showcase & Y & Rog & $\mathrm{M}$ & M & 3.2 & 3.0 & 3.3 \\
\hline ХРН 3073 & $\mathrm{~B}$ & Asg & M & M & 3.2 & 3.0 & 3.3 \\
\hline ACX 93 CN 108 & $\mathrm{Y}$ & $\mathrm{AC}$ & M & M & 3.2 & 3.3 & 3.1 \\
\hline GSS 3732 & $\mathrm{Y}$ & $\operatorname{Rog}$ & MR & M & 3.2 & 2.5 & 3.9 \\
\hline First Star & $\mathrm{B}$ & Sdw & MR & M & 3.2 & 2.8 & 3.7 \\
\hline Snowbird & $\mathrm{W}$ & Rog & M & M & 3.2 & 3.0 & 3.4 \\
\hline Snow White & $\mathrm{W}$ & $\mathrm{HM}$ & M & M & 3.2 & 3.3 & 3.2 \\
\hline Summer Sweet 7710 & Y & $\mathrm{AC}$ & MR & M & 3.3 & 2.5 & 4.0 \\
\hline GSS 3615 & $\mathrm{Y}$ & $\operatorname{Rog}$ & MR & M & 3.3 & 2.5 & 4.0 \\
\hline Market Star & $\mathrm{B}$ & Sdw & M & M & 3.3 & 3.0 & 3.3 \\
\hline Moonshot & $\mathrm{W}$ & Sdw & MR & M & 3.3 & 2.8 & 3.8 \\
\hline Shimmer & $\mathrm{Y}$ & Asg & M & M & 3.3 & 3.0 & 3.6 \\
\hline Sch 30131 & Y & IFS & M & MR & 3.3 & 3.5 & 3.0 \\
\hline Frontier 3030 & $\mathrm{~W}$ & Asg & MR & M & 3.3 & 2.8 & 3.9 \\
\hline Snowy Sun & B & $\operatorname{Rog}$ & MR & M & 3.3 & 2.8 & 3.9 \\
\hline \multirow[t]{2}{*}{ Confection } & $\mathrm{B}$ & $\mathrm{HM}$ & M & M & 3.3 & 3.3 & 3.4 \\
\hline & & & & & \multicolumn{3}{|c|}{ (continued on next page } \\
\hline
\end{tabular}

a Bayesian LSD $(k=100)=1.20$ in 1994 and 1.22 in 1996.

b $\mathrm{Y}=$ yellow, $\mathrm{W}=$ white, and $\mathrm{B}=$ bicolored.

c $\mathrm{AC}=$ Abbott and Cobb, Asg = Asgrow Seed Co., $\mathrm{Cr}=$ Crookham Co., FM = Ferry Morse Seed Co., HM = Harris Moran Seed Co., IFS = Illinois Foundation Seed, Rog = Rogers Seed Co., and Sdw = Seedway.

${ }^{\mathrm{d}} \mathrm{R}=$ resistant, $\mathrm{MR}=$ moderately resistant, $\mathrm{M}=$ moderate, $\mathrm{MS}=$ moderately susceptible, and $\mathrm{S}=$ susceptible.

e BLB ratings on a scale of 1 to 9: $1=$ chlorotic flecks; $3=$ most symptoms 3 to $10 \mathrm{~cm}$ long and followed leaf veins; $5=$ most symptoms 15 to $30 \mathrm{~cm}$ long; $7=$ most symptoms $50 \mathrm{~cm}$ or longer; and 9 = symptoms appear systemic on inoculated leaves. 
The experimental design was a split-plot randomized complete block with four replicates. Hybrids were planted in main plots, and BLB treatments were applied to subplots. Each experimental unit was a four- row plot. Rows were $3.6 \mathrm{~m}$ long and spaced $76 \mathrm{~cm}$ apart. Thirty-two kernels were planted two per hill on 17 May 1994 and 21 May 1996. Plants were thinned to 1 per hill (16 per row) on 9 June 1994 and 24 June 1996.

Table 1. (continued from previous page)

\begin{tabular}{|c|c|c|c|c|c|c|c|}
\hline \multirow[b]{2}{*}{ Hybrid } & \multirow{2}{*}{$\begin{array}{c}\text { Kernel } \\
\text { color }^{b}\end{array}$} & \multirow{2}{*}{$\begin{array}{c}\text { Seed } \\
\text { co. }^{c}\end{array}$} & \multicolumn{2}{|c|}{ Reaction to $\mathbf{B L B}^{\mathrm{d}}$} & \multicolumn{3}{|c|}{ Severity rating ${ }^{\mathrm{e}}$} \\
\hline & & & 1994 & 1996 & $\bar{x}$ & 1994 & 1996 \\
\hline ХРН 3074 & $\mathrm{~B}$ & Asg & MR & M & 3.3 & 2.3 & 4.4 \\
\hline Astro & $\mathrm{Y}$ & Sdw & MR & M & 3.4 & 2.8 & 4.0 \\
\hline Summer Sweet 8102 & $\mathrm{~B}$ & $\mathrm{AC}$ & M & M & 3.4 & 3.3 & 3.6 \\
\hline Hudson & B & Rog & M & M & 3.4 & 3.0 & 3.9 \\
\hline Honey n Pearl & B & IFS & M & M & 3.5 & 3.5 & 3.4 \\
\hline Summer Sweet 7612 & B & $\mathrm{AC}$ & MR & M & 3.5 & 2.5 & 4.4 \\
\hline Upstart & $\mathrm{Y}$ & $\mathrm{HM}$ & M & M & 3.5 & 3.8 & 3.2 \\
\hline BSS 3636 & B & $\operatorname{Rog}$ & $\mathrm{R}$ & $\mathrm{S}$ & 3.5 & 2.0 & 5.0 \\
\hline Candy Store & B & $\mathrm{HM}$ & MS & MR & 3.5 & 4.3 & 2.8 \\
\hline Landmark & Y & HM & MR & M & 3.5 & 2.8 & 4.3 \\
\hline BSS 4812 & $\mathrm{~B}$ & $\operatorname{Rog}$ & MR & M & 3.5 & 2.8 & 4.3 \\
\hline HM 701 & $\mathrm{Y}$ & $\mathrm{HM}$ & M & M & 3.5 & 3.8 & 3.3 \\
\hline ACX 92 CN 30 MDM & $\mathrm{Y}$ & $\mathrm{AC}$ & M & M & 3.6 & 4.0 & 3.1 \\
\hline Sch 5408 & B & IFS & M & M & 3.6 & 3.3 & 3.9 \\
\hline Aspen & $\mathrm{W}$ & Rog & M & M & 3.6 & 3.3 & 3.9 \\
\hline Zenith & $\mathrm{Y}$ & $\mathrm{HM}$ & M & M & 3.6 & 3.5 & 3.7 \\
\hline Top Notch & B & HM & M & M & 3.6 & 3.5 & 3.7 \\
\hline Princeton & B & Asg & MR & M & 3.6 & 2.8 & 4.4 \\
\hline Tribune & $\mathrm{Y}$ & $\mathrm{Cr}$ & $\mathrm{M}$ & M & 3.6 & 3.3 & 4.0 \\
\hline Crisp n Sweet 730BC & B & $\mathrm{Cr}$ & MS & MR & 3.6 & 4.3 & 3.0 \\
\hline HMX 2347 WS & $\mathrm{W}$ & HM & MR & MS & 3.7 & 2.8 & 4.6 \\
\hline ХРН 3056 & $\mathrm{Y}$ & Asg & M & M & 3.7 & 3.8 & 3.7 \\
\hline Diablo & B & $\mathrm{FM}$ & M & M & 3.7 & 3.5 & 3.9 \\
\hline GSS 4644 & $\mathrm{Y}$ & Rog & M & M & 3.7 & 4.0 & 3.4 \\
\hline GSS 6278 & $\mathrm{Y}$ & Rog & MS & M & 3.7 & 4.3 & 3.2 \\
\hline Summer Sweet 7620 & $\mathrm{Y}$ & $\mathrm{AC}$ & M & M & 3.7 & 3.3 & 4.2 \\
\hline GSS 3814 & $\mathrm{Y}$ & Rog & MS & M & 3.7 & 4.3 & 3.2 \\
\hline GSS 9239 & $\mathrm{Y}$ & Rog & MR & MS & 3.8 & 2.8 & 4.8 \\
\hline Sweeter Bi Far & $\mathrm{B}$ & $\mathrm{HM}$ & M & M & 3.8 & 4.0 & 3.6 \\
\hline How Sweet It Is & W & $\mathrm{Cr}$ & MS & M & 3.8 & 4.3 & 3.3 \\
\hline Harlequin & B & FM & $\mathrm{M}$ & M & 3.8 & 3.5 & 4.1 \\
\hline GSS 4369 & $\mathrm{Y}$ & $\operatorname{Rog}$ & M & MS & 3.8 & 3.4 & 4.8 \\
\hline XPH 3085 & B & Asg & M & M & 3.9 & 3.8 & 4.0 \\
\hline Shaker & $\mathrm{Y}$ & Asg & M & MS & 3.9 & 3.0 & 4.8 \\
\hline Treasure & W & IFS & MS & M & 4.0 & 4.3 & 3.7 \\
\hline Pro Sweet 213 R & $\mathrm{Y}$ & $\mathrm{AC}$ & M & M & 4.0 & 4.0 & 4.0 \\
\hline Supersweet Jubilee & $\mathrm{Y}$ & $\operatorname{Rog}$ & $\mathrm{S}$ & M & 4.0 & 4.5 & 3.6 \\
\hline Fortune & $\mathrm{Y}$ & IFS & M & MS & 4.0 & 3.5 & 4.6 \\
\hline BSS 4494 & B & Rog & $\mathrm{S}$ & M & 4.0 & 4.5 & 3.6 \\
\hline Sweetear & $\mathrm{Y}$ & $\mathrm{FM}$ & M & M & 4.1 & 4.0 & 4.1 \\
\hline Paradise & $\mathrm{Y}$ & $\operatorname{Rog}$ & M & $\mathrm{S}$ & 4.1 & 3.0 & 5.1 \\
\hline WSS 3680 & $\mathrm{~W}$ & $\operatorname{Rog}$ & $\mathrm{S}$ & M & 4.1 & 4.5 & 3.7 \\
\hline Summer Sweet 7100 & $\mathrm{Y}$ & $\mathrm{AC}$ & M & MS & 4.1 & 3.5 & 4.8 \\
\hline Dazzle & B & Asg & $\mathrm{S}$ & M & 4.2 & 4.8 & 3.6 \\
\hline WSS 4233 & W & Rog & M & MS & 4.2 & 3.8 & 4.6 \\
\hline HMX 2345 BS & $\mathrm{B}$ & HM & M & M & 4.2 & 4.0 & 4.3 \\
\hline ACX 92 CN 16RR & $\mathrm{Y}$ & $\mathrm{AC}$ & M & $\mathrm{S}$ & 4.2 & 3.0 & 5.3 \\
\hline GSS 3710 & $\mathrm{Y}$ & $\operatorname{Rog}$ & M & MS & 4.2 & 4.0 & 4.5 \\
\hline Crisp n Sweet 725 & $\mathrm{Y}$ & $\mathrm{Cr}$ & M & MS & 4.2 & 3.8 & 4.7 \\
\hline Florida Staysweet & $\mathrm{Y}$ & IFS & MS & M & 4.2 & 4.3 & 4.2 \\
\hline Paksweet & $\mathrm{Y}$ & FM & $\mathrm{S}$ & M & 4.3 & 4.5 & 4.0 \\
\hline Even Sweeter & W & Asg & $\mathrm{S}$ & M & 4.3 & 5.3 & 3.3 \\
\hline Honey Queen & $\mathrm{Y}$ & $\operatorname{Rog}$ & $\mathrm{S}$ & M & 4.3 & 4.8 & 3.9 \\
\hline Quest & B & Rog & M & $\mathrm{S}$ & 4.3 & 3.3 & 5.4 \\
\hline Escalade & B & $\mathrm{HM}$ & M & MS & 4.4 & 3.8 & 4.8 \\
\hline Royal Sweet & $\mathrm{Y}$ & $\operatorname{Rog}$ & $\mathrm{S}$ & M & 4.4 & 4.8 & 4.0 \\
\hline BSS 4544 & B & $\operatorname{Rog}$ & $\mathrm{S}$ & M & 4.4 & 4.5 & 4.3 \\
\hline ХРН 3086 & $\mathrm{~B}$ & Asg & $\mathrm{S}$ & M & 4.4 & 4.8 & 4.1 \\
\hline Primetime & $\mathrm{Y}$ & $\operatorname{Rog}$ & M & $\mathrm{S}$ & 4.4 & 4.0 & 4.9 \\
\hline Discovery & $\mathrm{Y}$ & Sdw & M & $\mathrm{S}$ & 4.5 & 3.3 & 5.7 \\
\hline Kandy Kiss & $\mathrm{Y}$ & $\mathrm{FM}$ & MS & MS & 4.5 & 4.3 & 4.8 \\
\hline ACX 92 CN 33RR & $\mathrm{Y}$ & $\mathrm{AC}$ & MS & $\mathrm{S}$ & 4.6 & 4.3 & 4.9 \\
\hline Sunset & $\mathrm{Y}$ & FM & $\mathrm{S}$ & MS & 4.6 & 4.5 & 4.7 \\
\hline Pegasus & W & $\mathrm{Cr}$ & $\mathrm{S}$ & M & 4.6 & 5.3 & 3.7 \\
\hline Autumn Star & B & Sdw & $\mathrm{S}$ & M & 4.6 & 5.0 & 4.2 \\
\hline Forever & $\mathrm{Y}$ & Asg & $\mathrm{S}$ & MS & 4.6 & 4.5 & 4.8 \\
\hline Sweet Top & $\mathrm{Y}$ & $\operatorname{Rog}$ & $\mathrm{S}$ & $\mathrm{S}$ & 5.2 & 5.0 & 5.3 \\
\hline
\end{tabular}

Bacterial suspensions were sprayed with hand sprayers into whorls of plants in the middle two rows of inoculated treatments, as described for the hybrid evaluation, with three additional inoculations after irrigation on 16 and 24 June and 8 July 1994. Symptoms of BLB were rated on a plot basis, as described previously, on 22 July 1994 and 15 July 1996. Primary ears from 10 consecutive plants in each of the middle 2 rows of each plot were harvested at freshmarket maturity, about 21 days after the midsilk stage. Due to differences in hybrid maturity, harvest dates ranged from 8 to 25 August 1994 and from 11 to 27 August 1996. The weight of 20 ears and the number of marketable ears were measured from each plot. Ears were weighed before and after removing husk leaves. Most of the ears classified as nonmarketable had poor kernel fill in the tip end of the ear, i.e., "poor tip fill."

BLB severity ratings, ear weight, and percentage of marketable ears were analyzed by ANOVA. Waller-Duncan BLSD values were used to compare main effect means of hybrids or hybrid means within treatments depending on the significance of the interaction term.

Table 2. Reactions of 30 shrunken-2 sweet corn inbreds to bacterial leaf blight (BLB) in trials in 1994 and 1996

\begin{tabular}{|c|c|c|}
\hline Inbred & $\begin{array}{l}\text { Kernel } \\
\text { color }^{\mathrm{a}}\end{array}$ & $\begin{array}{l}\text { BLB severity } \\
\text { rating }\end{array}$ \\
\hline 59 & Y & 1.5 \\
\hline 42 & $\mathrm{Y}$ & 1.8 \\
\hline 50 & $\mathrm{Y}$ & 2.0 \\
\hline 52 & $\mathrm{Y}$ & 2.0 \\
\hline 24 & $\mathrm{Y}$ & 2.2 \\
\hline 06 & W & 2.3 \\
\hline 21 & $\mathrm{Y}$ & 2.3 \\
\hline 44 & $\mathrm{Y}$ & 2.3 \\
\hline 14 & W & 2.4 \\
\hline 56 & $\mathrm{Y}$ & 2.5 \\
\hline 02 & $\mathrm{Y}$ & 2.5 \\
\hline 45 & $\mathrm{Y}$ & 2.5 \\
\hline 69 & $\mathrm{Y}$ & 2.6 \\
\hline 01 & $Y$ & 2.7 \\
\hline 77 & W & 2.7 \\
\hline 41 & $\mathrm{Y}$ & 2.7 \\
\hline 55 & $\mathrm{Y}$ & 3.2 \\
\hline 70 & $\mathrm{Y}$ & 3.5 \\
\hline 94 & $\mathrm{Y}$ & 3.5 \\
\hline 58 & $\mathrm{Y}$ & 3.6 \\
\hline 47 & W & 3.6 \\
\hline 11 & W & 3.7 \\
\hline 63 & W & 3.9 \\
\hline 09 & $\mathrm{Y}$ & 3.9 \\
\hline 30 & $\mathrm{Y}$ & 3.9 \\
\hline 54 & W & 4.3 \\
\hline 92 & $\mathrm{Y}$ & 4.4 \\
\hline 80 & $\mathrm{Y}$ & 4.5 \\
\hline 57 & W & 5.0 \\
\hline 78 & $\mathrm{Y}$ & 5.7 \\
\hline \multicolumn{2}{|c|}{ Bayesian LSD $(k=100)$} & 0.98 \\
\hline
\end{tabular}

a $\mathrm{Y}=$ yellow and $\mathrm{W}=$ white.

b BLB ratings on a scale of 1 to 9: $1=$ chlorotic flecks; $3=$ most symptoms 3 to $10 \mathrm{~cm}$ long and followed leaf veins; $5=$ most symptoms 15 to $30 \mathrm{~cm}$ long; $7=$ most symptoms $50 \mathrm{~cm}$ or longer; and $9=$ symptoms appear systemic on inoculated leaves. 


\section{RESULTS}

Hybrid evaluations. BLB symptoms were evident about 1 week after inoculation. Symptoms typically were restricted to leaf tissue that had been in the whorl at the time of inoculation. Hybrids with the greatest resistance often had chlorotic fleck or pinpoint-type symptoms that did not elongate. A few lesions on some hybrids extended the entire length of the inoculated leaf. Lesions were typically 5 to $25 \mathrm{~cm}$ long. The density of lesions occurring in a banded pattern across the leaf also varied among hybrids and appeared to be associated closely with lesion length, although length and density of lesions were not measured. Lesions typically had very distinct borders and were limited strictly by leaf veins, although on certain hybrids the margins of lesions were ill defined and wavy.

BLB severity ratings for the entire trial averaged 3.3 and 3.7 , with standard deviations of 0.84 and 0.8, in 1994 and 1996, respectively. The trial by hybrid interaction term was significant in the combined ANOVA, so hybrids were compared within trials. Hybrid means ranged from 1.8 to 5.5, with a BLSD value of 1.20 in 1994 (Table 1). Hybrid means ranged from 1.9 to 5.7, with a BLSD value of 1.22 in 1996 .

Classification of hybrid reactions usually

Table 3. Reaction of 29 shrunken-2 sweet corn hybrids to bacterial leaf blight (BLB) in trials in 1994 and 1996

\begin{tabular}{|c|c|c|c|c|}
\hline \multicolumn{2}{|c|}{ Cross } & \multirow{2}{*}{$\begin{array}{c}\text { BLB severity } \\
\text { rating }^{\mathrm{a}}\end{array}$} & \multirow{2}{*}{$\begin{array}{l}\text { Mid-parent } \\
\text { value }^{\text {b }}\end{array}$} & \multirow[b]{2}{*}{ Difference } \\
\hline Seed parent & Pollen parent & & & \\
\hline 21 & 24 & 1.9 & 2.2 & -0.3 \\
\hline 42 & 52 & 2.1 & 1.9 & 0.2 \\
\hline 21 & 45 & 2.3 & 2.4 & -0.1 \\
\hline 21 & 06 & 2.4 & 2.3 & 0.1 \\
\hline 94 & 52 & 2.4 & 2.8 & -0.4 \\
\hline 09 & 41 & 2.5 & 3.3 & -0.8 \\
\hline 21 & 69 & 2.6 & 2.5 & 0.1 \\
\hline 59 & 30 & 2.7 & 2.7 & 0 \\
\hline 21 & 47 & 2.7 & 2.9 & -0.2 \\
\hline 21 & 02 & 2.8 & 2.4 & 0.4 \\
\hline 44 & 24 & 2.8 & 2.2 & 0.5 \\
\hline 50 & 54 & 2.8 & 3.1 & -0.4 \\
\hline 94 & 01 & 2.8 & 3.1 & -0.3 \\
\hline 41 & 30 & 2.9 & 3.3 & -0.4 \\
\hline 94 & 70 & 2.9 & 3.5 & -0.6 \\
\hline 14 & 01 & 3.1 & 2.6 & 0.6 \\
\hline 14 & 70 & 3.2 & 3.0 & 0.2 \\
\hline 44 & 01 & 3.3 & 2.5 & 0.8 \\
\hline 11 & 01 & 3.5 & 3.2 & 0.3 \\
\hline 21 & 54 & 3.5 & 3.3 & 0.2 \\
\hline 09 & 01 & 3.7 & 3.3 & 0.4 \\
\hline 01 & 92 & 3.7 & 3.5 & 0.2 \\
\hline 63 & 57 & 3.7 & 4.5 & -0.7 \\
\hline 41 & 80 & 3.9 & 3.6 & 0.3 \\
\hline 21 & 58 & 3.9 & 2.9 & 1.0 \\
\hline 09 & 30 & 4.0 & 3.9 & 0.1 \\
\hline 63 & 41 & 4.4 & 3.3 & 1.1 \\
\hline 55 & 56 & 4.8 & 2.9 & 1.9 \\
\hline 41 & 78 & 5.0 & 4.2 & 0.8 \\
\hline \multicolumn{2}{|c|}{ Bayesian LSD value $(k=100)$} & 0.98 & & \\
\hline
\end{tabular}

${ }^{a}$ BLB ratings on a scale of 1 to 9: $1=$ chlorotic flecks; $3=$ most symptoms 3 to $10 \mathrm{~cm}$ long and followed leaf veins; $5=$ most symptoms 15 to $30 \mathrm{~cm}$ long; $7=$ most symptoms $50 \mathrm{~cm}$ or longer; and $9=$ symptoms appear systemic on inoculated leaves.

${ }^{b}$ Arithmetic mean of rating for seed and pollen parents.

${ }^{\mathrm{c}}$ Difference between BLB severity rating and mid-parent values. noted for having severe or moderately severe symptoms in disease nurseries when BLB occurred naturally in Urbana, IL, from 1990 to 1993 . Mean BLB severity ratings were greater than 4 for 16 of the 32 hybrids, between 3 and 4 for 12 of the 32 hybrids, and less than 3 for 4 of the 32 hybrids.

Inbred and crosses. BLB severity ratings for 30 inbreds and 29 hybrids from crosses of the inbreds averaged 3.2 in 1994 and 3.1 in 1996. The main effect of trials and interaction terms, including trials, were not significant in the combined ANOVA, so combined data were analyzed. Reactions of inbreds ranged from 1.5 to 5.7 (Table 2). Reactions of hybrids ranged from 1.9 to 5 (Table 3). The slope coefficient of the regression of BLB severity ratings of crosses on mid-parent values was not different from $1\left(b_{1}=0.86 \pm 0.18\right.$; Fig. 1$)$. The relatively modest value for the coefficient of determination $\left(r^{2}=0.44\right)$ resulted primarily from a few crosses with BLB severity ratings different from mid-parent values. BLB severity ratings of $4.8,4.4$, and 3.9 for crosses of $55 \times 56,63 \times 41$, and $21 \times 58$ were 1.9 , 1.1 , and 1 greater than mid-parent values, respectively. Also, BLB severity ratings of 5.0 and 3.3 for the crosses of $41 \times 78$ and $44 \times 01$ were 0.8 greater than mid-parent values, and the 2.5 rating for $09 \times 41$ was 0.8 less than the mid-parent value.

Yield-loss trials. Mean BLB severity ratings for hybrids were compared within BLB treatments due to significant hybrid by treatment interaction terms in both the 1994 and 1996 trials (Tables 4 and 5). In inoculated plots, BLB severity ratings ranged from 2.3 to 5.1 in 1994 and from 2.3 to 5.0 in 1996. In noninoculated plots, BLB severity ratings ranged from 1.4 to 3.3 in 1994 and from 0.1 to 1.7 in 1996. Dry weather during June 1996 limited the spread of $A$. avenae subsp. avenae among plots, whereas wet weather during 1994 caused consider-

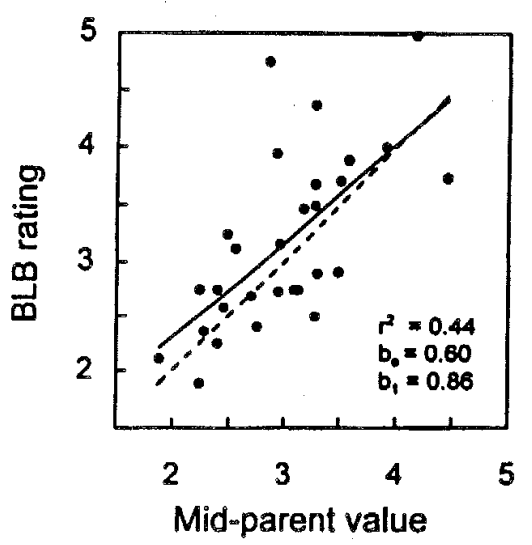

Fig. 1. Bacterial leaf blight (BLB) severity ratings for 29 crosses from 30 shrunken-2 sweet corn inbred lines and mid-parent values calculated as arithmetic means of inbreds. Coefficient of determination $\left(r^{2}\right)$, intercept $\left(b_{0}\right)$, and slope $\left(b_{1}\right)$ coefficients are from least squares linear regression of severity ratings of crosses on mid-parent values. Dashed line has a slope of 1 . 
able development of symptoms in noninoculated plots. Reactions of hybrids to BLB (e.g., ranks) in the yield-loss trial in 1994 were similar to those in the hybrid evaluation for 16 hybrids common to both studies; however, in 1996 ratings of 3.6 and 3.7 for inoculated plots of cvs. Summer Sweet 7630 and Punchline were higher than expected, and ratings of 2.5, 2.9, and 3.1 for inoculated plots of cvs. How Sweet It Is, Treasure, and Even Sweeter, respectively, were lower than expected compared to the hybrid evaluation.

Ear weights and percent marketable ears differed among hybrids in the 1994 trial, but there was no significant effect of BLB inoculations on either of these measures of yield (Table 4). Hybrid by treatment interactions were not significant. In the 1996 trial, marketable ears differed among hybrids, but the main effect of BLB treatments and the hybrid by treatment interaction were not significant. The hybrid by treatment interaction term was significant in the ANOVA of ear weight for the 1996 trial, so treatments were compared within hybrids.

Ear weights in inoculated plots in 1996 were about 10 to $15 \%$ lower than those from noninoculated plots for two hybrids, cvs. Even Sweeter and Pegasus (Table 5). Ear weights did not differ among BLB treatments for the other 10 hybrids. Differences in BLB severity ratings from inoculated and noninoculated plots of cvs. Even Sweeter and Pegasus were 3 and 2.8, respectively, which was the same as or less than the differences in severity ratings for six other hybrids for which yields were not affected by BLB.

\section{DISCUSSION}

Supersweet sh2 sweet corn hybrids varied considerably in their reactions to BLB. Resistant hybrids and inbreds often had chlorotic fleck symptoms that occurred in bands across leaves as a result of infection in the whorl. Susceptible hybrids had dense bands of lesions that often were $50 \mathrm{~cm}$ long. Despite severe foliar symptoms, BLB did not affect yield substantially in these trials, even among hybrids with the most susceptible reactions. Thus, symptom severity was not correlated with yield.

A previous study reported that BLB symptoms did not affect dry matter digestibility of field corn hybrids (6). These data indicate that BLB causes cosmetic damage to leaves, but little economic loss should occur unless conditions for infection are considerably greater than those in our trials or the trials in Georgia during the mid-1970s (6). The cosmetic injury caused by BLB on susceptible and moderately susceptible sweet corn hybrids could be important if husk leaves are severely infected; however, ears should be largely unaffected if levels of disease are similar to those in our trials. Thus, in most situations the economic consequence of severe BLB probably is limited to additional costs of removing severely symptomatic husk leaves for cosmetic purposes.

Groups of yellow, white, and bicolored hybrids did not differ in their reactions to BLB. Although there is no reason to expect that genes for kernel color affect reactions to BLB, we hypothesized that certain groups of related inbreds with white kernels might be very susceptible, based on anecdotal ob- servations in disease nurseries in Illinois and Florida. Our evaluations of 122 hybrids, 30 inbreds, and 29 hybrid crosses of the inbreds do not support this hypothesis. Resistant and susceptible reactions were observed among yellow and white inbreds and among yellow, white, and bicolored hybrids. Considering the aggregated distribution of BLB-infected plants and distinct disease gradients previously observed in

Table 4. Bacterial leaf blight (BLB) rating and yield of 24 shrunken-2 sweet corn hybrids evaluated in 1994

\begin{tabular}{|c|c|c|c|c|}
\hline \multirow[b]{2}{*}{ Hybrid } & \multicolumn{2}{|c|}{ BLB severity rating ${ }^{a}$} & \multirow{2}{*}{$\begin{array}{c}\text { Ear } \\
\text { weight }^{\mathbf{b}}(\mathrm{g})\end{array}$} & \multirow{2}{*}{$\begin{array}{r}\text { Marketable } \\
\text { ears }(\%)^{\mathrm{c}}\end{array}$} \\
\hline & Inoculated & Control & & \\
\hline Punchline & 2.3 & 1.6 & 206 & 99 \\
\hline Sch 5005 & 2.3 & 1.5 & 264 & 98 \\
\hline Summer Sweet 7702 & 2.4 & 1.8 & 209 & 85 \\
\hline Challenger & 2.5 & 1.6 & 232 & 91 \\
\hline Summer Sweet 7630 & 2.5 & 1.4 & 270 & 100 \\
\hline ХPH 3073 & 2.6 & 1.8 & 259 & 91 \\
\hline Summer Sweet 7210 & 2.7 & 1.6 & 231 & 100 \\
\hline Summer Sweet 8102 & 2.8 & 1.6 & 272 & 100 \\
\hline Sch 40134 & 3.2 & 1.9 & 271 & 99 \\
\hline Frontier & 3.3 & 2.3 & 229 & 80 \\
\hline Princetor & 3.4 & 1.8 & 255 & 94 \\
\hline Fortune & 3.5 & 2.1 & 302 & 100 \\
\hline Sch 5408 & 3.7 & 2.3 & 200 & 89 \\
\hline Quest & 3.7 & 2.4 & 233 & 94 \\
\hline Primetime & 3.8 & 2.4 & 233 & 96 \\
\hline Moonshot & 3.9 & 2.4 & 248 & 96 \\
\hline Dazzle & 4.2 & 2.8 & 244 & 96 \\
\hline Snow White & 4.3 & 2.8 & 229 & 88 \\
\hline Even Sweeter & 4.5 & 2.6 & 229 & 92 \\
\hline Sch 30131 & 4.5 & 2.6 & 250 & 98 \\
\hline Summer Sweet 8701 & 4.9 & 2.8 & 214 & 98 \\
\hline How Sweet It Is & 4.9 & 3.0 & 219 & 88 \\
\hline Pegasus & 5.0 & 2.8 & 183 & 92 \\
\hline Treasure & 5.1 & 3.3 & 242 & 99 \\
\hline Bayesian LSD $(k=100)$ & 0.94 & 0.51 & 44.8 & 12.9 \\
\hline
\end{tabular}

a BLB ratings on a scale of 1 to 9: $1=$ chlorotic flecks; $3=$ most symptoms 3 to $10 \mathrm{~cm}$ long and followed leaf veins; $5=$ most symptoms 15 to $30 \mathrm{~cm}$ long; $7=$ most symptoms $50 \mathrm{~cm}$ or longer; and $9=$ symptoms appear systemic on inoculated leaves.

${ }^{\mathrm{b}}$ Inoculated with Acidovorax avenae subsp. avenae.

${ }^{\mathrm{c}}$ Percentage of primary ears that were marketable.

Table 5. Bacterial leaf blight (BLB) ratings and yield of 12 shrunken-2 sweet corn hybrids evaluated in 1996

\begin{tabular}{|c|c|c|c|c|c|}
\hline \multirow[b]{2}{*}{ Hybrid } & \multicolumn{2}{|c|}{ BLB severity rating ${ }^{\mathrm{a}}$} & \multicolumn{2}{|c|}{ Ear weight (g) } & \multirow{2}{*}{$\begin{array}{l}\text { Marketable } \\
\text { ears }(\%)^{\mathbf{b}}\end{array}$} \\
\hline & Inoculated $^{\mathrm{c}}$ & Control & Inoculated & Control $^{\mathrm{d}}$ & \\
\hline Sch 5005 & 2.3 & 0.4 & 311 & 308 & 98 \\
\hline How Sweet It Is & 2.5 & 0.3 & 261 & 271 & 88 \\
\hline Treasure & 2.9 & 0.7 & 263 & 278 & 100 \\
\hline Even Sweeter & 3.1 & 0.1 & $256^{\mathrm{e}}$ & $280^{\mathrm{e}}$ & 98 \\
\hline Frontier & 3.2 & 0.8 & 257 & 276 & 75 \\
\hline Snow White & 3.2 & 0.3 & 235 & 247 & 91 \\
\hline Summer Sweet 7630 & 3.6 & 0.3 & 273 & 293 & 91 \\
\hline Punchline & 3.7 & 0.1 & 227 & 226 & 95 \\
\hline Pegasus & 3.7 & 0.9 & $228^{\mathrm{e}}$ & $269^{\mathrm{e}}$ & 88 \\
\hline Dazzle & 4.1 & 0.3 & 275 & 267 & 82 \\
\hline Quest & 4.6 & 1.7 & 331 & 324 & 92 \\
\hline Fortune & 5.0 & 1.2 & 220 & 209 & 75 \\
\hline Bayesian LSD $(k=100)$ & 0.70 & 0.75 & 25.7 & 25.2 & 22.3 \\
\hline
\end{tabular}

${ }^{a}$ BLB ratings on a scale of 1 to 9: $1=$ chlorotic flecks; $3=$ most symptoms 3 to $10 \mathrm{~cm}$ long and followed leaf veins; $5=$ most symptoms 15 to $30 \mathrm{~cm}$ long; $7=$ most symptoms $50 \mathrm{~cm}$ or longer; and $9=$ symptoms appear systemic on inoculated leaves.

${ }^{\mathrm{b}}$ Percentage of primary ears that were marketable.

${ }^{\mathrm{c}}$ Inoculated with Acidovorax avenae subsp. avenae.

${ }^{\mathrm{d}}$ Noninoculated control treatment.

${ }^{\mathrm{e}}$ Ear weight from inoculated treatment significantly less than ear weight from noninoculated control treatment. 
fields of naturally infected sweet corn (1), it should not be surprising that ratings from trials in which BLB occurs naturally may not produce an accurate representation of hybrid reactions. In fact, only one-half of the hybrids with severe or moderately severe symptoms in disease nurseries with natural infection were rated greater than 4 in our inoculated trials, whereas $38 \%$ were rated from 3 to 4 and $12 \%$ were rated less than 3 .

Mid-parent values gave a relatively good indication of hybrid reactions to BLB, with the exception of a few specific hybrid crosses, most of which tended to be more severely infected than expected based on mid-parent values. A more complete set of crosses is needed to determine the gene action controlling these reactions. Several hybrids and inbreds had chlorotic fleck or resistant reactions. These materials could be used to improve the BLB resistance of susceptible inbreds, although our evaluation of inbreds and their crosses suggests that this resistance may not have a high degree of dominance. Considering the sporadic occurrence of BLB and the apparent lack of significant economic impact of this disease, improving sh2 sweet corn for resistance to BLB probably should remain a low priority in most breeding programs.

\section{ACKNOWLEDGMENTS}

We thank B. Stall, University of Florida, Gainesville, for isolates of $A$. avenae subsp. avenae used as inoculum and Abbott and Cobb Inc., Asgrow Seed Company, Crookham Company, Ferry Morse Seed Company, Harris Moran Seed Company, Illinois Foundation Seed Inc., Rogers Seed Company, and Seedway Inc. for sweet corn seed used in these studies.

\section{LITERATURE CITED}

1. Gitaitis, R. D., Stall, R. E., and Strandberg, J. O. 1978. Dissemination and survival of Pseudo- monas albopercipitans ascertained by disease distribution. Phytopathology 68:227-231.

2. Gitaitis, R. D., Stall, R. E., and Strandberg, J. O. 1981. Electron microscopy of the ingress and establishment of Pseudomonas albopercipitans in sweet corn leaves. Phytopathology 71:171-175.

3. Johnson, A. G., Robert, A. L., and Cash, L. 1949. Bacterial leaf blight and stalk rot of corn. J. Agric. Res. 78:719-732.

4. Lopes, C. A., and Stall, R. E. 1990. Biological control of Pseudomonas avenae with epiphytic bacteria isolated from corn plants. Pesqui. Agropecu. Bras. 25:1125-1131.

5. Smith, D. R., and White, D. G. 1988. Diseases of corn. Pages 687-766 in: Corn and Corn Improvement. 3rd ed. G. F. Sprague and J. W. Dudley, eds. American Society of Agronomy, Madison, WI.

6. Sumner, D. R., and Schaad, N. W. 1977. Epidemiology and control of bacterial leaf blight of corn. Phytopathology 67:1113-1118.

7. White, D. G., Pataky, J. K., and Stall, R. E. 1994. Unusual occurrences of bacterial leaf blight on maize and sorghum in central Illinois. Plant Dis. 78:640. 